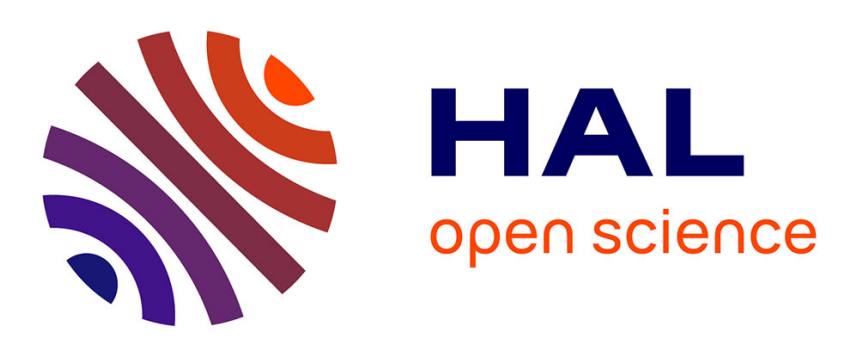

\title{
Numerical simulation of a counter-rotative open rotor using phase-lagged conditions. Initial validation on a single rotor case
}

\author{
Maxime Fiore, Romain Biolchini
}

\section{- To cite this version:}

Maxime Fiore, Romain Biolchini. Numerical simulation of a counter-rotative open rotor using phaselagged conditions. Initial validation on a single rotor case. Journal of Turbomachinery, 2020, 142 (12), pp.121002-121011. 10.1115/1.4047891 . hal-03318218

\author{
HAL Id: hal-03318218 \\ https://hal.science/hal-03318218
}

Submitted on 9 Aug 2021

HAL is a multi-disciplinary open access archive for the deposit and dissemination of scientific research documents, whether they are published or not. The documents may come from teaching and research institutions in France or abroad, or from public or private research centers.
L'archive ouverte pluridisciplinaire HAL, est destinée au dépôt et à la diffusion de documents scientifiques de niveau recherche, publiés ou non, émanant des établissements d'enseignement et de recherche français ou étrangers, des laboratoires publics ou privés. 


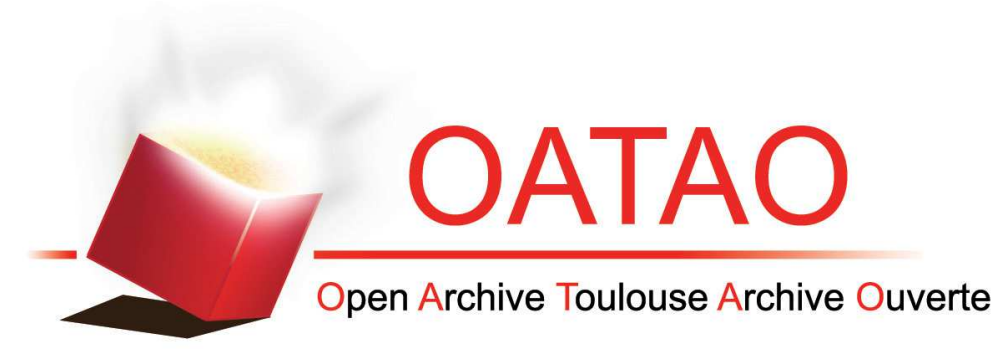

\section{Open Archive Toulouse Archive Ouverte (OATAO)}

OATAO is an open access repository that collects the work of some Toulouse researchers and makes it freely available over the web where possible.

This is an author's version published in: https://oatao.univ-toulouse.fr/27946

Official URL : https://doi.org/10.1115/1.4047891

\section{To cite this version :}

Fiore, Maxime and Biolchini, Romain Numerical simulation of a counter-rotative open rotor using phase-lagged conditions. Initial validation on a single rotor case. (2020) Journal of Turbomachinery, 142 (12). 121002-121011. ISSN 0889-504X

Any correspondence concerning this service should be sent to the repository administrator: tech-oatao@listes-diff.inp-toulouse.fr 


\section{Numerical simulation of a counter-rotative open rotor using phase-lagged conditions. Initial validation on a single rotor case}

\author{
Maxime Fiore* \\ CFD team CERFACS \\ Toulouse, France \\ Email: fiore@cerfacs.fr
}

\author{
Romain Biolchini \\ Safran Aircraft Engines \\ Moissy-Cramayel, France \\ Email: romain.biolchini@safrangroup.com
}

\begin{abstract}
This paper presents the Large Eddy Simulation (LES) of a propeller representative of the first rotor of a Counter Rotative Open Rotor (CROR) configuration based on a multiple frequency phase-lagged approach in conjunction with a Proper Orthogonal Decomposition (POD) data storage. This method enables to perform unsteady simulations on multistage turbomachinery configurations including multiple frequency flows with a reduction of the computational domain composed of one single blade passage for each row. This approach is advantageous when no circumferential periodicity occurs in the blade rows of the configuration and a full $360^{\circ}$ simulation would be required. The data storage method is based on a POD decomposition replacing the traditional Fourier Series Decomposition (FSD). The inherent limitation of phase-shifted periodicity assumption remains with POD data storage but this compression method alleviates some issues associated to the Fourier transform, especially spectrum issues. The paper is first dedicated to compare the flow field obtained with the LES with phaselagged condition against full-matching URANS, LES simulations and experimental data available around the blade and in the wake of the rotor. The study shows a close agreement of the phase-lagged LES simulation with other simulations performed and a thicker wake compared to the experiments with lower turbulent activity. The analysis of the losses generated in the configuration, based on an entropy formulation and a splitting between boundary layer and secondary flow structures, shows the strong contribution of the blade boundary layer in the losses generated.
\end{abstract}

\section{Introduction}

In order to decrease fuel consumption and greenhouse gases emissions of airplanes propulsion systems, new engines with significantly higher By-Pass Ratios (BPR) compared to current turbofan engines are being developed as Counter Rotative Open Rotor (CROR). Due to their unducted architecture, new acoustic issues emerge since the noise emitted is not damped by the nacelle like in classical turbofan [1]. Also, the blading design and geometrical arrangement of these engines [2] considerably modify the flow topology and the mechanisms of loss generation compared to turbofan architectures [3].

The development of such new aircraft engine designs has been widely performed numerically especially through the use of Unsteady Reynolds Averaged Navier-Stokes (URANS) simulations [4-9]. Turbulent processes as tip vortex interaction, rotor wakes, transition processes on blades may limit the applicability of (U)-RANS approaches where all turbulent structures are modelled. This observation pushes towards the use of lower levels of turbulence modelling as Large Eddy Simulation (LES) approach [10] where the large energetic scales of turbulence are resolved and only the small scales are modelled. The use of LES simulation in aircraft engine configurations is still challenging since the requirements for the meshing, numerics, convergence and data extraction are generally higher compared to (U)-RANS. In addition, the simulation domain cannot be reduced using periodic boundary conditions due to a coprime number of blades for the two counter rotative rows. In order to alleviate the cost of a full $360^{\circ}$ simulation, some paths have been investigated to reduce the computational cost of LES, with acceptable physical restrictions. In that regard, the use of phase-lagged boundary conditions allows the $360^{\circ}$ configuration to be reduced to a single passage per row configuration, as proposed by Erdos and Alzner [11]. The main difficulty with such conditions consists in storing the flow over one period at the phase-lagged interfaces (in multi-row simulations, it consists in storing the information at the interface between static and rotating domains and on lateral azimuthal conditions). For the meshes and time steps considered in practical turbomachinery simulations, the direct storage represents a significant cost. The most popular method to reduce the memory cost is to store only the coefficients of the Fourier Series Decomposition (FSD) of the temporal signal, as proposed by He $[12,13]$. The FSD is truncated to a limited number of harmonics, and the coefficients are updated

\footnotetext{
*Address all correspondence to this author.
} 
each time step with the shape correction method. This method assumes that the flow is perfectly periodic in time, which is a fair assumption in URANS for operating points dominated by periodic rotor-stator interactions (wakes and potential effects). For LES simulation, the periodic assumption is no longer true. In particular, LES includes multiple unknown frequencies, for example non deterministic small scales structures in a turbulent wake, for which the characteristic time that needs to be used is unknown. Keeping this limitation of the phase-lagged assumption, a data storage based on a Proper Orthogonal Decomposition (POD) [14] has been developed and implemented in the elsA software [15] by Mouret et al. [16] used to perform the present LES simulation. This compression method especially improves some issues associated to the Fourier decomposition and the corresponding spectrum content.

The purpose of this paper is to assess the capability of LES simulation to properly recover the flow in a propeller similar to first rotor row of a CROR configuration, estimate the impact of phase-lagged conditions with POD reconstruction and study the related losses generated. The first part of this paper introduces the configuration and numerical set up. Two full-matching periodic simulations are performed based on a URANS and LES formalisms, and a LES simulation with phase-lagged conditions that are compared against experiments. The LES approach is then used to describe the physical mechanisms and related losses in the configuration.

\section{Configuration and numerical methods}
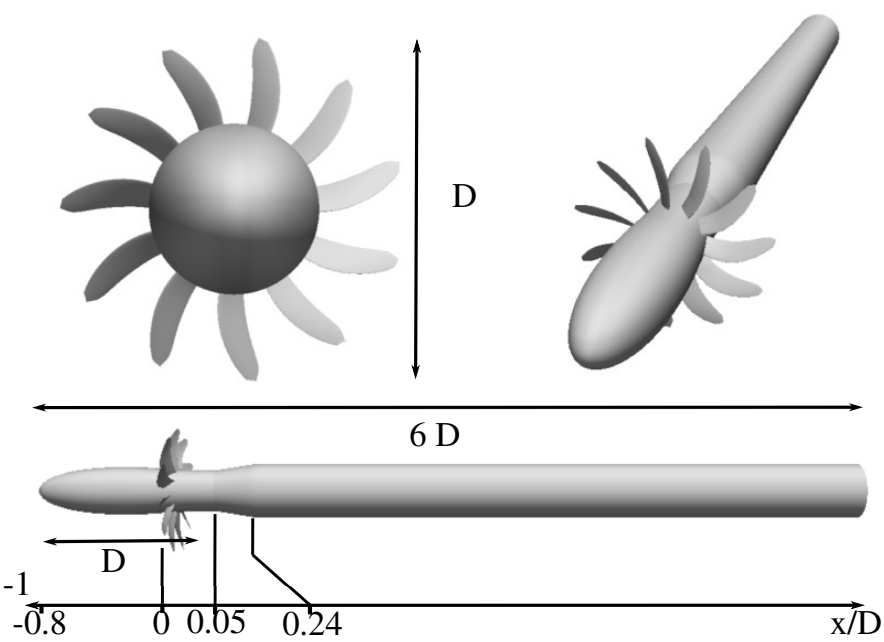

Fig. 1: Geometrical set up features

Th experimental set-up is composed of a nacelle with a rotor row representative of the upstream rotor of a full CROR configuration designed by Airbus at scale 1/7 [17]. The main geometrical features are shown in Fig. 1 where the characteristic dimensions are expressed in terms of the rotor diameter D. This configuration has been implemented and tested in the Airbus low-speed wind-tunnel in Bremen, Germany by
Table 1: Characteristics of the experimental set up

\begin{tabular}{cccc}
\hline \multicolumn{2}{c}{ cascade details } & \multicolumn{2}{c}{ nominal conditions } \\
\hline Casing radius/D & 0.17 & $p_{\text {tot, } \infty}[\mathrm{Pa}]$ & 100986 \\
Tip radius/D & 0.5 & $T_{\text {tot, } \infty}[\mathrm{K}]$ & 311.5 \\
$\begin{array}{c}\text { Forward nacelle } \\
\text { length/D }\end{array}$ & 1 & $\begin{array}{l}\text { Rot. speed } \\
\omega\left[\mathrm{rad}^{-1} \mathrm{~s}^{-1}\right.\end{array}$ & 663.2 \\
Nacelle length/D & 5 & $\operatorname{Re}_{\mathrm{D}}$ & $1.45 \times 10^{6}$ \\
Blade number & 11 & $\mathrm{Ma}_{\infty}$ & 0.2 \\
\hline
\end{tabular}

the DLR (see Fig. 2). The experimental measurements are composed of oil-painting visualizations around the blades and Particle Image Velocimetry (PIV) measurements downstream of the blade [18]. Upstream conditions and rotational speed of the rotor row used in the experiments are gathered in Tab. 1.

In order to prepare the simulation of a full CROR configuration using LES and phase-lagged conditions, three simulations are performed and compared against experiments. Two full-matching periodic configurations are simulated based on URANS and LES formalisms and a LES with phase-lagged conditions. Since the set-up is equipped with one rotor row composed of 11 blades, 1/11 of the full domain is considered in the full-matching URANS and LES simulations and periodic conditions are applied on the lateral surfaces. These two simulations are performed by taking into account that the relatively large scale structures in the flow may be affected by the azimuthal periodic conditions. Also, for the LES simulation, since the fundamental assumption of the direct periodicity is that the solutions at each passage are the same even though they are in a different position, some care is given to the small scale non deterministic unsteady structures for example in the wake that may face frequency shift at the periodic boundary condition. The computational domain is split in two parts at the axial position of the hypothetical/future rotor/rotor interface of a full CROR configuration. The first part contains one passage of the front row extending from the inlet to the rotor/rotor interface. The second one is empty with the same azimuthal angle and extends from the rotor/rotor interface to the outlet of the domain. A full-matching condition is applied at the interface between the two subdomains (see Fig. 3). The corresponding URANS and LES simulations are denoted URANS M.P. $_{\text {. }}$ and LES $_{\text {M.P. }}$ where the subscript stands for matching periodic. The LES with phase-lagged conditions and POD data storage is performed over a different simulation domain. The domain of simulation is also split in two subdomains: the upstream domain is identical to the one used for the reference simulation and a larger downstream subdomain (1/9 of the whole domain) rotating in opposite direction to the upstream subdomain. This computational domain mimics an hypothetical/future downstream counter-rotative rotor row with nine blades of a CROR configuration. This simulation is denoted LES $_{\text {P.L. }}$ where the subscript stands for phase-lagged. The 


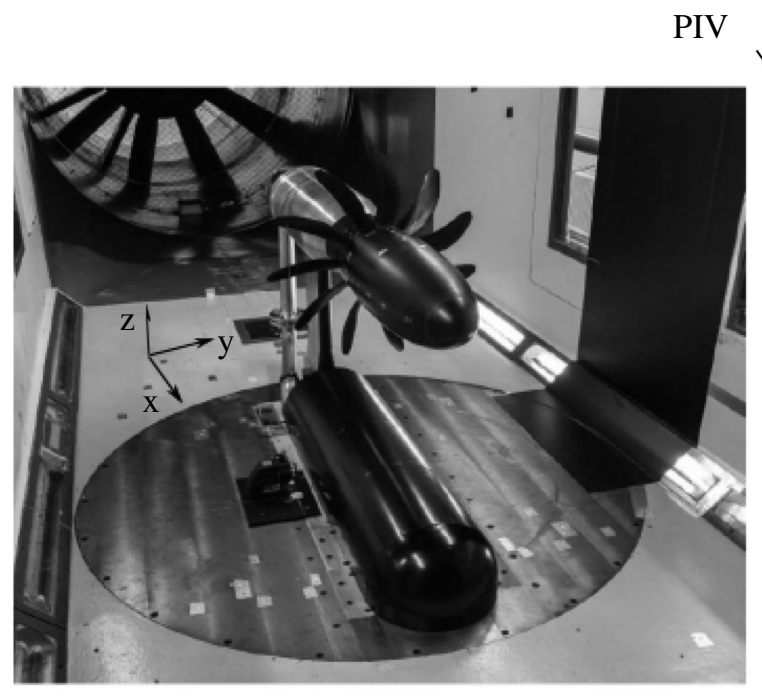

(a)

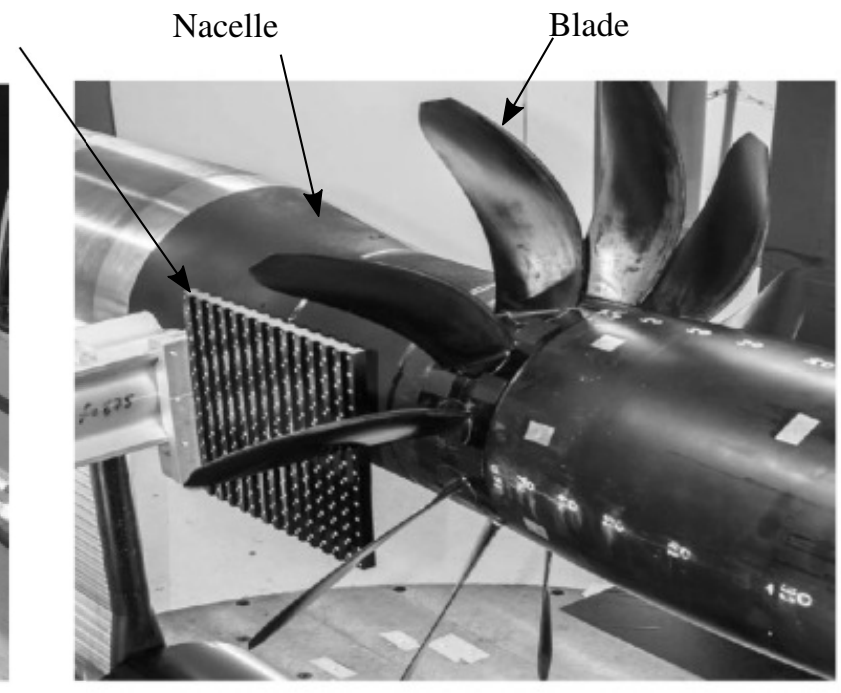

(b)

Fig. 2: Experimental set-up [18] with axis definition (a) and PIV measurements for the wake analysis (b)

Table 2: Data compression rate CR with POD data storage ( $\mathrm{W}_{\mathrm{POD}}$ ) compared to a full data storage over one blade passage $\mathrm{w}_{\text {direct }}=10.3 \mathrm{Go}$

\begin{tabular}{ccc}
\hline $\begin{array}{c}\text { Number of POD } \\
\text { modes }\end{array}$ & $\begin{array}{c}\text { Data storage } \\
\text { WPOD }(G o)\end{array}$ & $\begin{array}{c}\text { Compression } \\
\text { rate CR }\end{array}$ \\
\hline 10 & 1.2 & 0.88 \\
20 & 2.5 & 0.76 \\
30 & 3.8 & 0.63 \\
\hline
\end{tabular}

simulation domain for all the simulations extends $4 \mathrm{D}$ upstream of the configuration in the streamwise direction. The outlet is set at the rear of the nacelle which is $5 \mathrm{D}$ long. The radius of the simulation domain is $3.5 \mathrm{D}$.

The boundary conditions imposed at the free surfaces are extrapolation conditions of the reference state (see Tab. 1), which is also used as an initial solution for the simulation. Adiabatic and non-slipping wall conditions are applied on the blade and nacelle walls. For the LES $_{\text {P.L. }}$, at the full non-matching interface and on the lateral surfaces, phase-lagged conditions are applied while full-matching interface and periodic conditions for the lateral sections are applied in the URANS ${ }_{\text {M.P. }}$ and LES $_{\text {M.P. }}$. The number of POD modes used to perform the data compression is set to 30 based on the sensitivity analysis proposed in next Sec. 3.1. The data compression rate $\mathrm{CR}=1-\mathrm{wPOD}_{\mathrm{PO}} / \mathrm{w}_{\text {direct }}$ of the POD method $\mathrm{W}_{\mathrm{POD}}$ compared to the direct storage memory cost $\mathrm{w}_{\text {direct }}$ for different number of POD modes is shown in Tab. 2.

The meshing is based on a $\mathrm{O}-6 \mathrm{H}$ block strategy (see Fig. 4 for the blade mesh refinement). The first off-wall point is set to $\mathrm{y}_{1} / \mathrm{C}_{x}=4 \times 10^{-5}$. Figure 5 shows $\mathrm{y}_{1}^{+}$distribution around the blade and nacelle, $y_{1}^{+}$remains below 10 on the nacelle surface and 2 over the blade surface. According to the

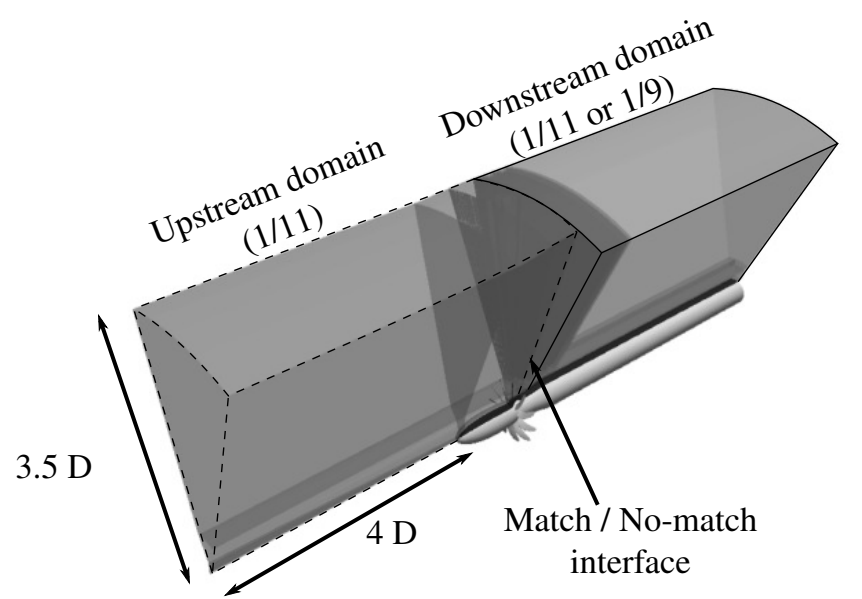

Fig. 3: Simulation domain. Matching interface for the URANS $_{\text {M.P., }}$ LES $_{\text {M.P. }}($ domain 1/11-1/11) and no-match interface for the LES $_{\text {P.L. }}$ (domain 1/11-1/9)

literature [19-21], the near-wall region should be resolved with $50 \leq \Delta x^{+} \leq 80 ; y_{1}^{+} \leq 1 ; 15 \leq \Delta z^{+} \leq 25$. These criteria are challenging to fulfil for the present configuration with relatively high Reynolds number and large dimensions. In wall-resolved LES, the streaks that are long and flow aligned structures close to the wall [22] are important structures to be resolved. These structures are shown to have lower variation in the $\mathrm{x}$ direction compared to $\mathrm{y}$ and $\mathrm{z}$ directions. Therefore, the mesh has been mainly coarsened in the $\mathrm{x}$ direction. In the spanwise direction, around 600 layers have been set over a spanwise length of around $15 \mathrm{~cm}$ with a grid refinement close to the nacelle wall (expansion ratio of 1.05). This provides a mean spanwise coordinate of $z^{+} \simeq 60$. In the stream direction, around 300 points have been set for a mid chord length of $10 \mathrm{~cm}$ that corresponds to a mean streamwise coordinate of $x^{+} \simeq 100$. The mesh is build to have an orthogonality 

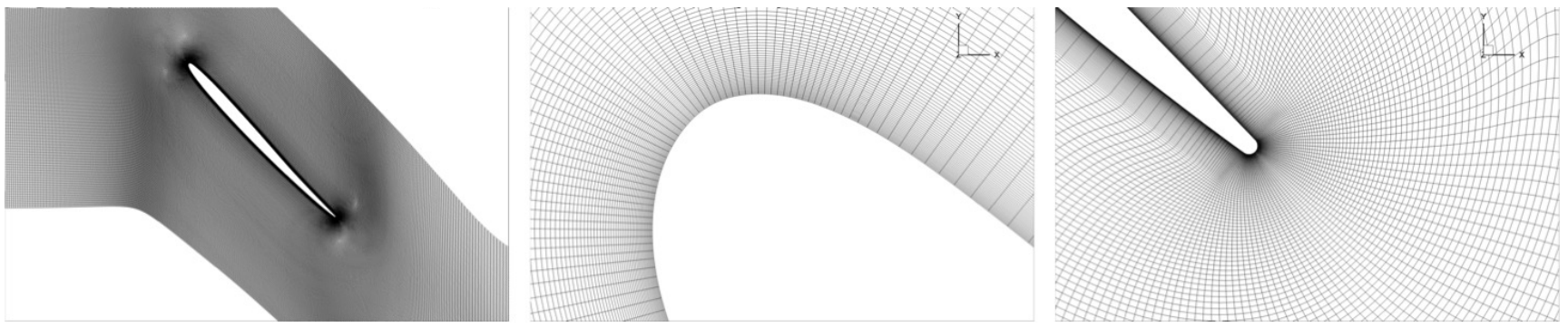

Fig. 4: Meshing around the blade, at the leading edge and trailing edge

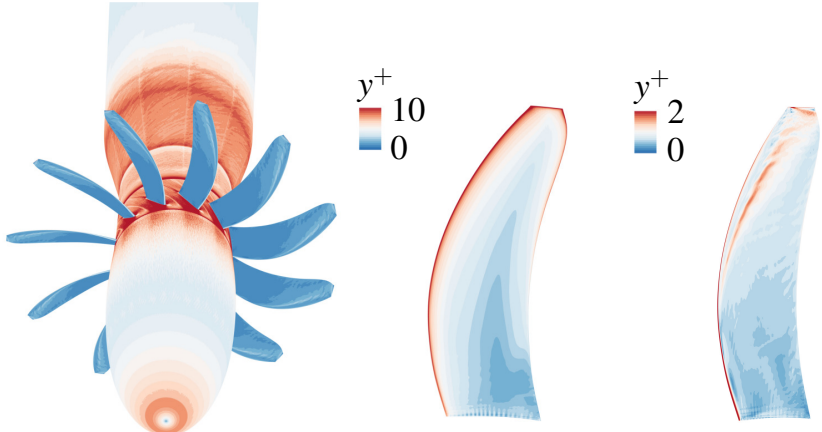

Fig. 5: $y_{1}^{+}$resolution on the nacelle and blades $\left(\mathrm{LES}_{\mathrm{P} . \mathrm{L} .}\right)$

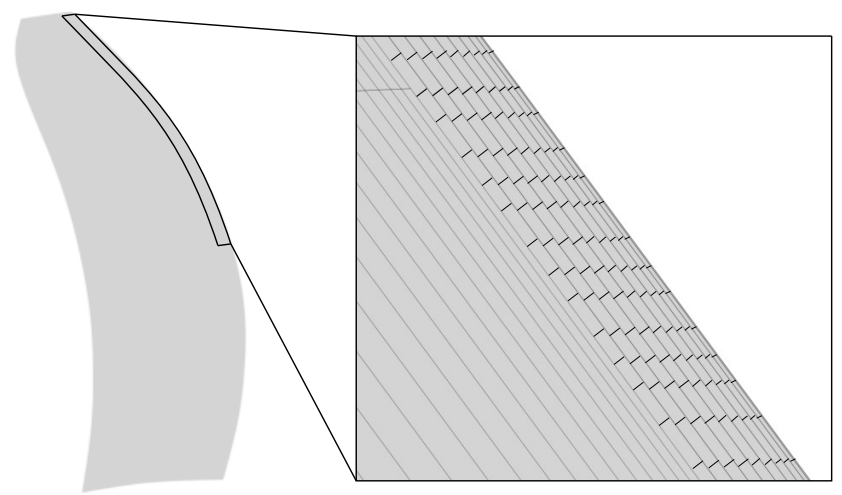

Fig. 6: Blade suction side tripping at the leading edge

higher than $30^{\circ}$ and in near-wall region higher than $80^{\circ}$. The stretching ratio between the size of neighbouring cells in the blade wall-normal direction was set to 1.03 to ensure around 30 grid points in the viscous layer until $y^{+}=50$. The mesh is refined in the wake to properly propagate the structures developing at the trailing edge of the blade. For the characteristic Reynolds number of the configuration $\left(\operatorname{Re}_{\mathrm{D}}=1.45 \times 10^{6}\right)$ and due to non negligible free-stream turbulence level in the experiments (around $\mathrm{Tu}=0.5-1 \%$ ), based on previous experiments of Abu-Ghanam and Shaw [23], the boundary layer on the blade suction side is expected to become turbulent close to the blade leading edge. In the LES simulations, since homogeneous inlet conditions are applied at the inlet and to prevent any natural transition process that would not have occurred in the experiments, a tripping is applied at the blade suction side leading edge to enforce transition process.
This technique consists in deforming the grid with randomly placed Gaussian profiles with maximum height variation of $0.8 \mathrm{~mm}$ (see Fig. 6). The tripping on the blade leading edge with a step has been used in a similar numerical study based on the NASA SDT configuration by Casalino et al. [24]. The mesh is composed of around $250 \times 10^{6}$ cells for the $\mathrm{LES}_{\mathrm{M} . P \text {. }}$ and $270 \times 10^{6}$ cells LES $_{\text {P.L. }}$. The URANS U.P. simulation is performed with the same mesh as the $\mathrm{LES}_{\text {M.P. }}$ except that no tripping is applied on the blade.

The simulations are performed using the ONERA elsA

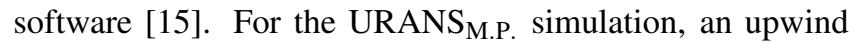
Roe scheme with third-order limiter [25] is used for the convective terms. The equivalent turbulent viscosity is obtained from the Wilcox k- $\omega$ two-equations model with Zheng's limiter [26]. For the LES simulations, a second order centred scheme with a low Jameson artificial viscosity [27] $\left(\kappa_{\mathrm{jam}}^{4}=0.002\right)$ is used for the convective discretization. The subgrid scale model is the Wall-Adapting Local Eddyviscosity (WALE) [28]. The time step is adapted to mesh resolution close to the wall $\Delta t^{+}=\Delta t \quad \mathrm{u}_{\infty} / \mathrm{C}_{x}=1 \times 10^{-5}$ (i.e. 11880 iteration per full rotation). Time integration is performed using Dual Time Stepping (DTS) with a CrankNicholson scheme (second order accurate) in combination with implicit pseudo-time stepping for the inner loops [29].

\section{Numerical/experimental comparison}

\subsection{One dimensional quantity comparison}

The convergence of the mass flow rate $\dot{m}$ at the interface between upstream and downstream domains and the thrust produced by the rotor $F_{\mathrm{T}}$ are monitored for the URANS $\mathrm{U}_{\mathrm{M} . P \text {. }}$ and LES $_{\text {P.L. }}$ simulations. The two quantities are expressed in terms of their respective coefficients $C_{\mathrm{M}}$ and $C_{\mathrm{T}}$ defined as:

$$
\begin{gathered}
C_{\mathrm{M}}=\frac{\dot{m}}{\rho_{\infty} \mathrm{D}^{3} \omega} \\
C_{\mathrm{T}}=\frac{F_{\mathrm{T}}}{\rho_{\infty} \mathrm{D}^{4} \omega^{2}} .
\end{gathered}
$$

The mass flow rate and thrust coefficients reach a constant value after around 10 full rotations (see Fig. 7 and 8). The thrust coefficient obtained with the LES P.L. matches well the value measured in the experiments $C_{\mathrm{T}}=0.0104(816 \mathrm{~N})$ with 


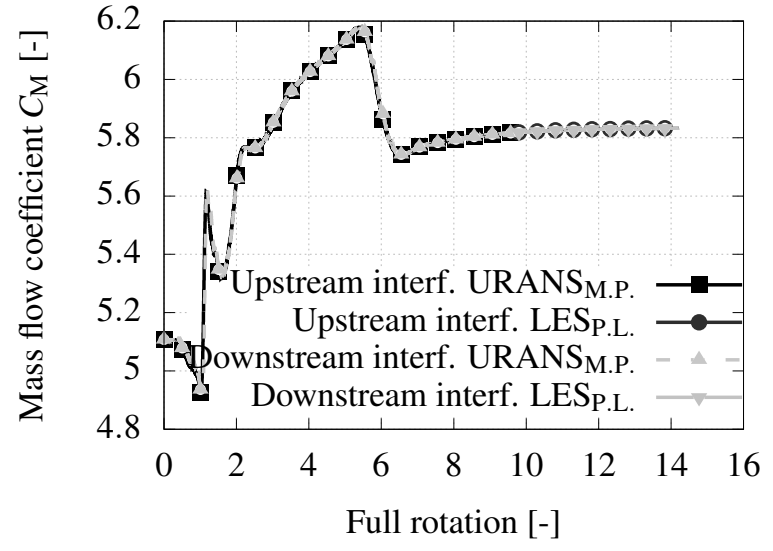

Fig. 7: Convergence of mass flow rate at the interface between upstream and downstream domains for the $\mathrm{URANS}_{\mathrm{M} . P .}$ and $\mathrm{LES}_{\mathrm{P} . \mathrm{L} \text {. }}$

a discrepancy of around $0.1 \%$. The influence of the number of POD modes used for the data compression has also been assessed by comparing the mass flow and thrust coefficient for different number of modes stored (3, 10, 20, 30 and 40, see Fig. 9). The results show that for a number of POD modes higher than 30 , the mass flow and thrust coefficients are relatively constant which is in accordance with a previous study from Mouret et al. [16]. In the present study, 30 POD modes are used for the data compression.

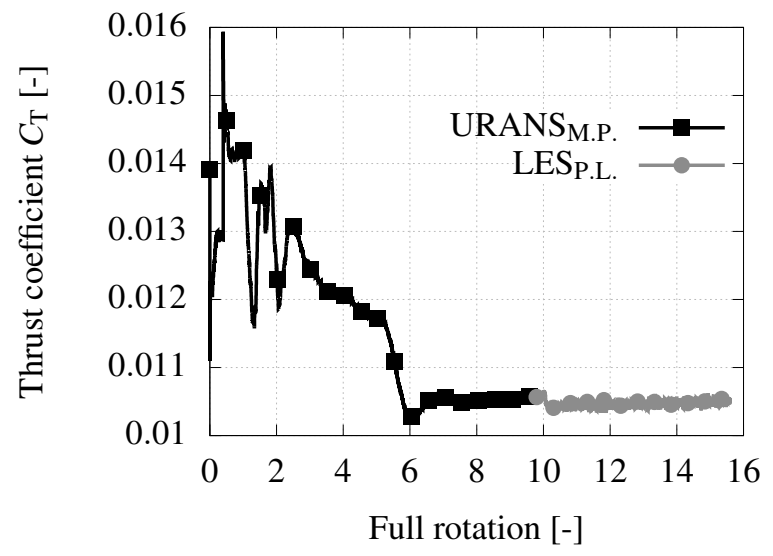

Fig. 8: Convergence of thrust for URANS M.P. and LES $_{\text {P.L. }}$

\subsection{Boundary layer state comparison}

Based on the time-averaged flow field obtained from the simulations and the boundary layer edge detection method available in elsA $[30,31]$, the boundary layer thickness $\delta$ and characteristic near-wall quantities can be obtained. The shape factor $H=\delta / \theta$ where $\delta$ and $\theta$ are the boundary layer displacement and momentum thickness is used to characterize the boundary layer nature in the simulations and compare

M. Fiore et al.

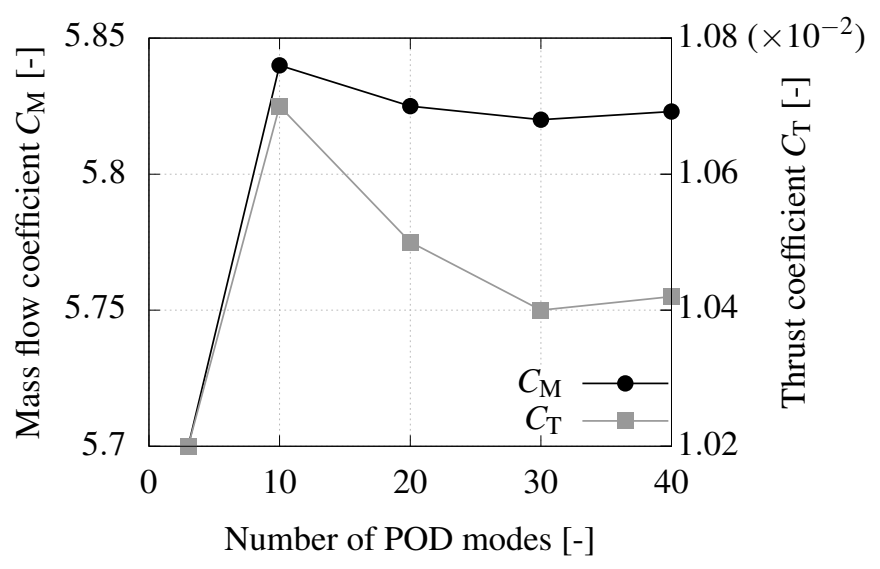

Fig. 9: Convergence of the mass flow and thrust coefficients depending on the number of POD modes used for data compression

it against experiments. Based on the Blasius profile, i.e., solution of the steady two-dimensional Prandtl equation in laminar regime, the value for $\mathrm{H}$ corresponding to laminar flows is 2.59 while 1.3 to 1.4 is typical of turbulent flows ${ }^{1}$ [32].

In the experiments, the boundary layer state on the blade suction side is based on oil-painting visualizations. Dark regions correspond to the regions where the oil-painting has been removed and may be associated to a turbulent boundary layer since the friction at wall is higher compared to a laminar one. On the contrary, light areas correspond to regions where the oil-painting is maintained and may be associated to a laminar boundary layer. On the blade suction side, the boundary layer is mainly laminar until midspan in a toothshape pattern. On the upper part of the blade, the boundary layer is fully turbulent from the blade leading edge (see Fig. 10 right). In the LES simulation without tripping, on the upper part of the blade, the boundary layer is laminar until around $10 \%$ chord where it becomes turbulent. The transition process is caused by the leading edge vortex which, in turn, is due to the blade sweep. Compared to straight blades, the sweeping of the blades reduces the normal Mach number and the shock losses for flight Mach number above 0.6 similarly to swept wing of commercial aircraft. This induces the development of a leading edge vortex similarly to the one observed on delta wings for example. Under high loading, this vortex merges with the tip vortex and is responsible for the localized separation bubble close to the leading edge that induce the transition to turbulence from midspan to the blade tip. In the tripped configuration, the boundary layer becomes turbulent close to the blade leading edge which is in better agreement with the experiments (see Fig. 10 left). Also, the tripping promotes a fully turbulent boundary layer and prevent from any transition processes and potential bursts released that could mix downstream with the wake. Some dis-

\footnotetext{
${ }^{1}$ This criterion based on the flow over a flat plate without pressure gradient is considered a good approximation in the configuration since the curvature of the nacelle and the blade surface is low. Moreover, the flow is relatively two-dimensional except in secondary vortices regions at the junction between nacelle and blade and the pressure gradients are moderate.
} 

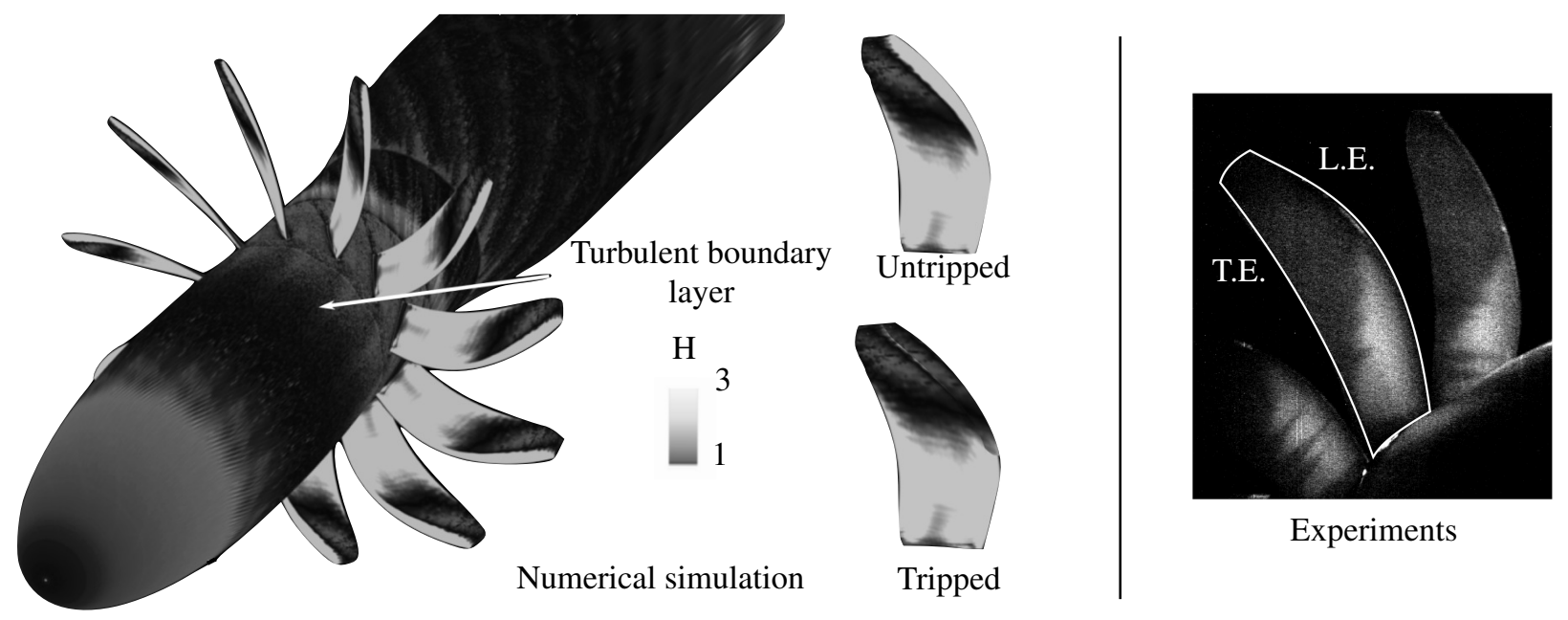

Experiments

Fig. 10: Shape factor for the untripped and tripped blade leading edge for the LES $_{\text {P.L. }}$ (left) and experimental oil visualization on the blade suction side (right). Light regions correspond to laminar boundary layer and dark ones to turbulent

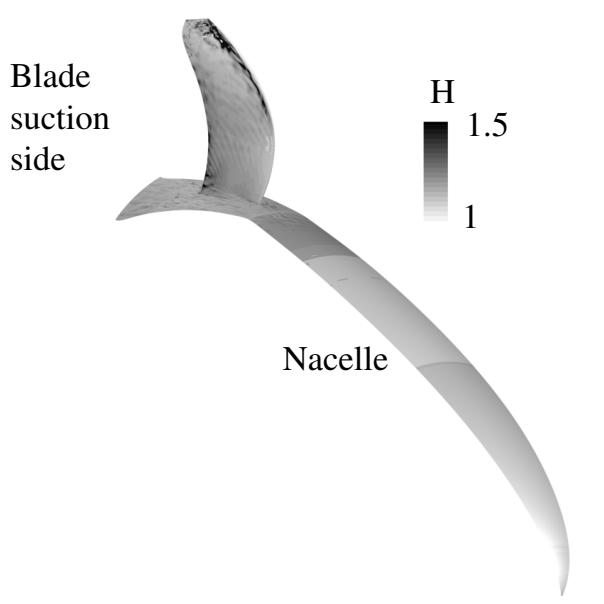

Fig. 11: Shape factor for the URANS M.P. simulation

crepancy can be still observed with the experiments since the turbulent region is oriented downwards on the blade suction side in the experiments while oriented upwards in the numerical simulation. For the URANS M.P. simulation, the shape factor is restricted to values between 1 and 1.5 (see Fig. 11). Except at the blade suction side near the leading edge where the shape factor is relatively high and thus the momentum close to the wall is relatively low, the shape factor is around 1.2-1.4 characterizing a turbulent boundary layer all over the blade.

\subsection{Wake profile comparison}

The comparison of the numerical simulations against experimental data is focused on the rotor wake. The velocity field downstream of the rotor is measured experimentally with a PIV system [33]. PIV measurements are performed along a square plane of $100 \mathrm{~mm} \times 100 \mathrm{~mm}$ set downstream of the rotor row at a constant radius ( $69 \%$ of blade span) in an $(\mathrm{X}, \mathrm{Z})$ plane. The velocity profiles are extracted over a line orthogonal to the wake. The comparison against experiments of the URANS $\mathrm{UR}_{\text {M.P. }}, \mathrm{LES}_{\text {M.P. }}$ and $\mathrm{LES}_{\mathrm{P} . \mathrm{L} \text {. results are }}$ compared with experimental data for a line $0.12 \mathrm{~m}$ downstream of the leading edge (see Fig. 12 center for the measurement plane location). The comparison for the mean axial velocity and fluctuation is shown in Fig. 13 and 14. The URANS $_{\text {M.P. }}$ LES M.P. and LES $_{\text {P.L. show a good agreement }}$ with the experiments for the mean velocity magnitude out of the wake. In the wake region characterized by the velocity deficit, the wake is thicker for the URANS M.P. and the two LES compared to the experiments. Also the velocity deficit is lower compared to the experiments with a discrepancy around $10 \%$. A similar trend is observed on the axial velocity fluctuation with a larger wake and lower velocity fluctuations of numerical simulations compared to the experiments. The LES $P$.L. shows however a lower turbulent activity compared to the $\mathrm{LES}_{\mathrm{M} . \mathrm{P} \text {. }}$. The POD data storage is able to reduce data filtering compared to Fourier data storage but part of the wake structure is inherently lost due to the phase-lagged assumption has been noticed by Mouret et al. [16]. This filtering process can be observed in Fig. 12 where some structures of the wake are lost when crossing interfaces (dashed lines). The larger wake with lower velocity deficit observed in the simulations can be associated to a thicker boundary layer at the trailing edge compared to the experiments. This phenomenon may be promoted by a too coarse grid in near-wall region and in the wake despite the efforts made to approach the near-wall refinement requirements in LES.

The comparison against experiments have shown that LES is able to match the thrust generated by the experimental configuration and a similar near-wall flow behaviour. Despite potential thicker boundary layer in the simulation that lead to a thicker wake compared to the experiments, sufficient confidence can be given in the numerical simulation to be used to describe the losses generated in this configuration. 

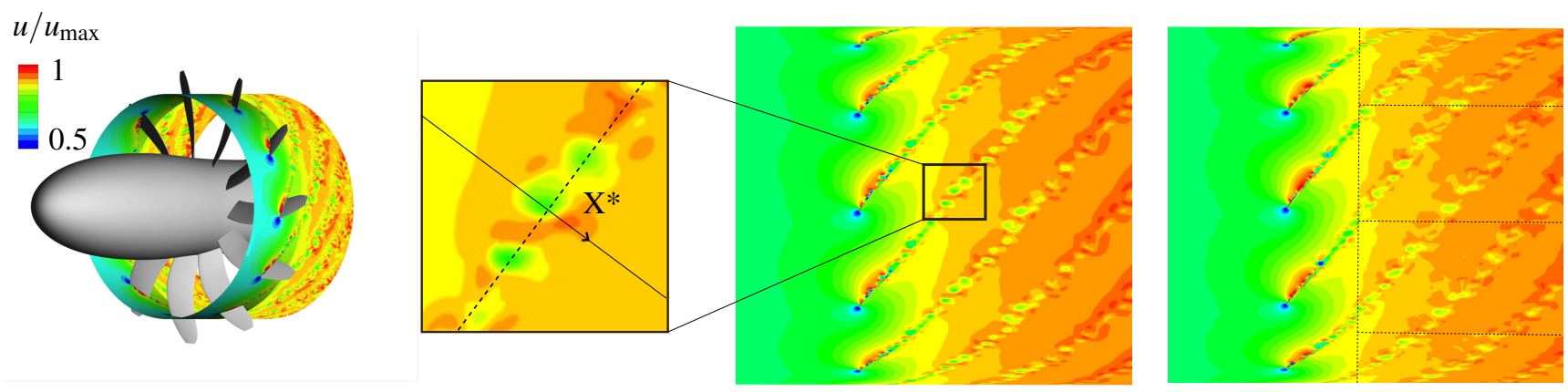

Fig. 12: Axial velocity along the simulation domain for the LES $_{\text {M.P. }}$ (center) and LES $_{\text {P.L. }}$ (right) including PIV measurement location (left)

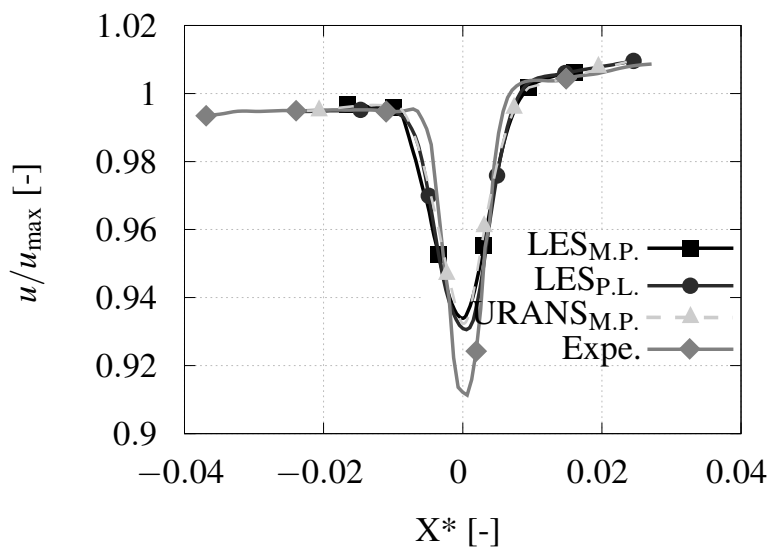

Fig. 13: Comparison of experimental and numerical results for mean axial velocity

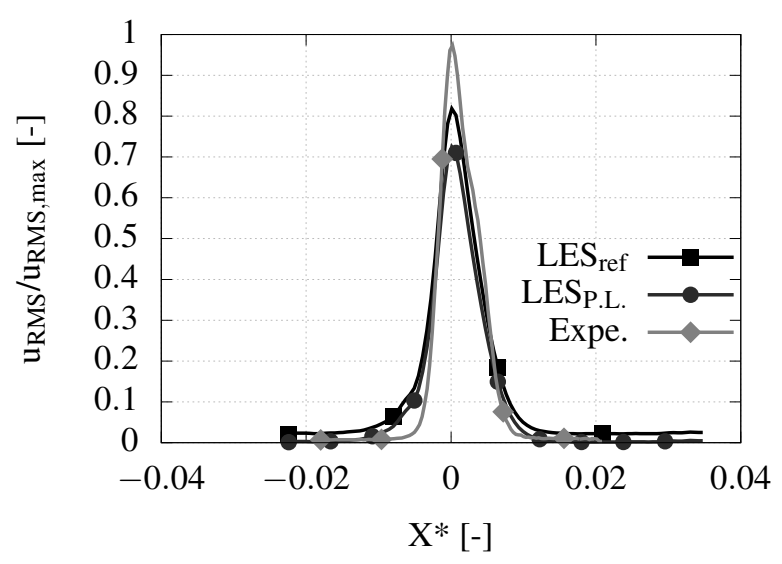

Fig. 14: Comparison of experimental and numerical results for fluctuating velocity

\section{Loss generated in the simulation domain}

The losses generated in the configuration are based on the volumetric entropy generation S. The entropy is the sum of two components: viscous dissipation and thermal diffu- sion [34] which are given by eq. (3) and (4) [35-39]:

$$
\begin{gathered}
S_{\text {visc }}=\widetilde{\widetilde{\tau_{i j, \text { eff }}} \frac{1}{\widetilde{T}} \frac{\partial \widetilde{u_{i}}}{\partial x_{j}}} \\
S_{\text {therm }}=\overline{\left(\lambda+\lambda_{\text {SGS }}\right) \frac{1}{\widetilde{T}^{2}}\left(\frac{\partial \widetilde{T}}{\partial x_{j}}\right)^{2}}
\end{gathered}
$$

where $\tau_{i j, \text { eff }}=\left(\mu+\mu_{\mathrm{SGS}}\right)\left(\partial \widetilde{u}_{i} / \partial x_{j}+\partial \widetilde{u_{j}} / \partial x_{i}\right)$ is the effective viscous stress tensor, $\mu_{\mathrm{SGS}}$ and $\lambda_{\mathrm{SGS}}$ are the equivalent subgrid scale viscosity and diffusion, . refers to the filtered quantity and . to the temporal averaging operator. These quantities can be integrated over axial subdomains of characteristic length $\mathrm{dx}$ to observe the evolution of irreversibilities along the domain. The corresponding evolution of the viscous and

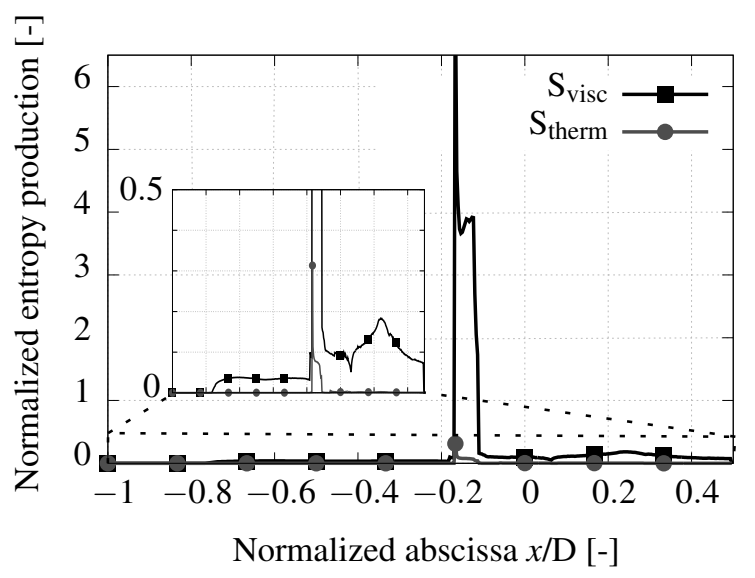

Fig. 15: Viscous and thermal entropy production along the simulation domain with a zoomed view

thermal entropy production along the domain are shown in Fig. 15. By integrating the area under the two curves, the 


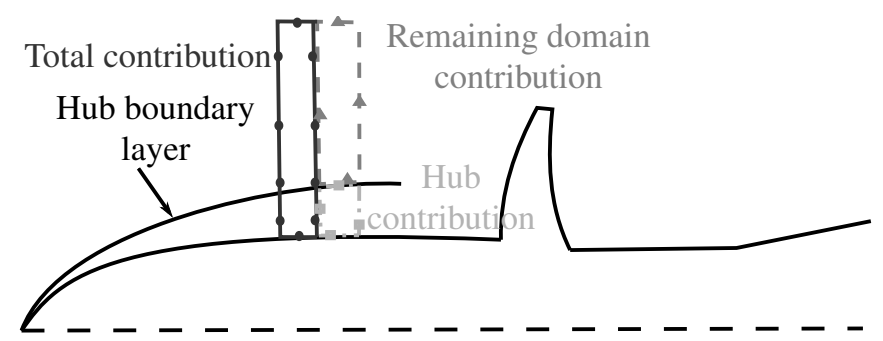

Fig. 16: Simulation domain discretized in axial subvolumes (dark grey). This subvolume can be split into two subvolumes: the nacelle boundary layer (light grey) and its complement that is the whole subvolume less the subvolume associated to the nacelle boundary layer (medium grey)

contribution of viscous and thermal gradients on the generation of loss can be assessed. In the current configuration without any inlet temperature inhomogeneity, no heat transfer at the wall, the thermal contribution is around two orders of magnitude lower than the viscous one. Since the thermal contribution is very low compared to the viscous contribution, the following analysis is focused on the viscous contribution. Based on the boundary layer thickness,

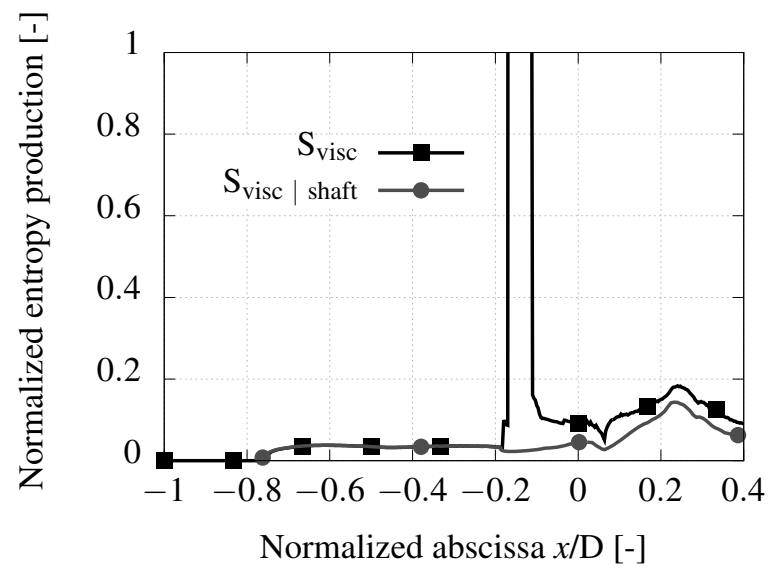

Fig. 17: Total viscous entropy production and contribution restricted to the nacelle boundary layer

the corresponding three-dimensional domain is extracted for the nacelle and blade and used to extract the corresponding boundary layer contributions in the generation of losses (see Fig. 16). The contribution of nacelle boundary layer to viscous loss generation is shown in Fig. 17. Negligible losses are generated upstream of the configuration for $x / D<-0.8$. Between $x / D=-0.8$ and $x / D=-0.17$, the losses generated are associated to the nacelle boundary layer contribution where the wall-normal velocity gradients generate losses. Along and downstream of the blade extent, the nacelle boundary layer contribution to losses increases due to a boundary layer becoming thicker with the axial position considered. Also, the steep increase associated to the nacelle boundary layer between $x / D=0.05$ and $x / D=0.24$

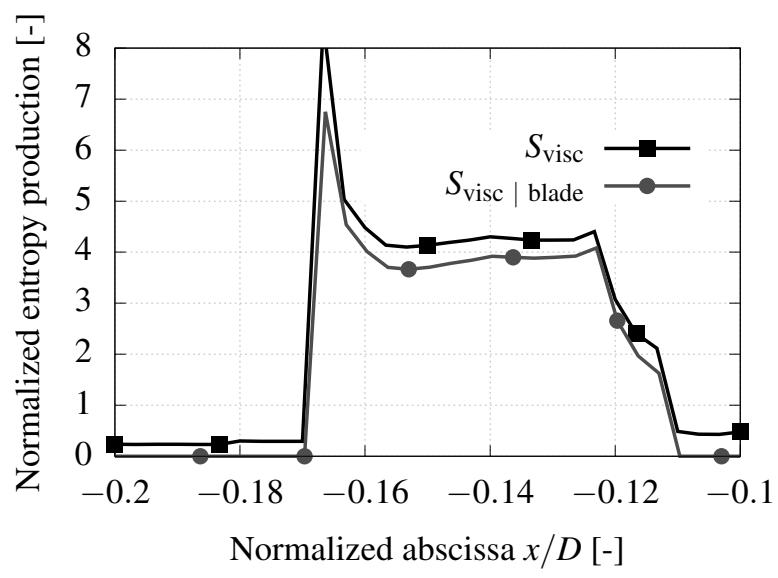

Fig. 18: Total viscous entropy production and contribution restricted to the blade boundary layer, the axial axis is restricted around the blade extent

is attributed to the diverging shape of the nacelle promoting larger wetted surface.

The contribution of the blade boundary layer to the losses generated is shown in Fig. 18. The boundary layer represents around $90 \%$ of the losses generated along the extent of the blade between $x / D=-0.17$ and -0.11 . This contribution is the main source of losses in the configuration and is in agreement with previous studies [40,41].

When the boundary layer contributions are subtracted to the total amount of loss generated, one can extract the remaining domain contribution. This contribution can be associated to the different vortices developing in the configuration: the passage vortex induced by the separation of the nacelle boundary layer and the cross-pressure gradient between two adjacent blades, the turbulent wake downstream of the trailing edge and the tip vortex (see Fig. 19). This contribution is almost negligible upstream of the blade leading edge. The contribution increases along the blade extent and close to the trailing edge due to the passage/tip vortex development and turbulent wake then decrease downstream of the blade characterizing the dissipation of theses vortical structures (see Fig. 20).

\section{Conclusion}

The numerical simulation of a propeller representing the front rotor of a CROR configuration has been performed. A total of three simulations have been carried out: two simulations with a full matching interface and periodic boundary conditions applied to the lateral surfaces in URANS and LES formalisms and a third simulation (LES) based on a phaselagged approach with POD data storage.

The simulations showed a good matching with the thrust generated by the propeller in the experiments. Also, the suction side boundary layer state for the different simulations agrees well with the experiments on the top part of the blade since the URANS simulation enforces a turbulent boundary layer similarly to the experiments and the tripping set in LES simulations promote a quick transition to turbulence close to 


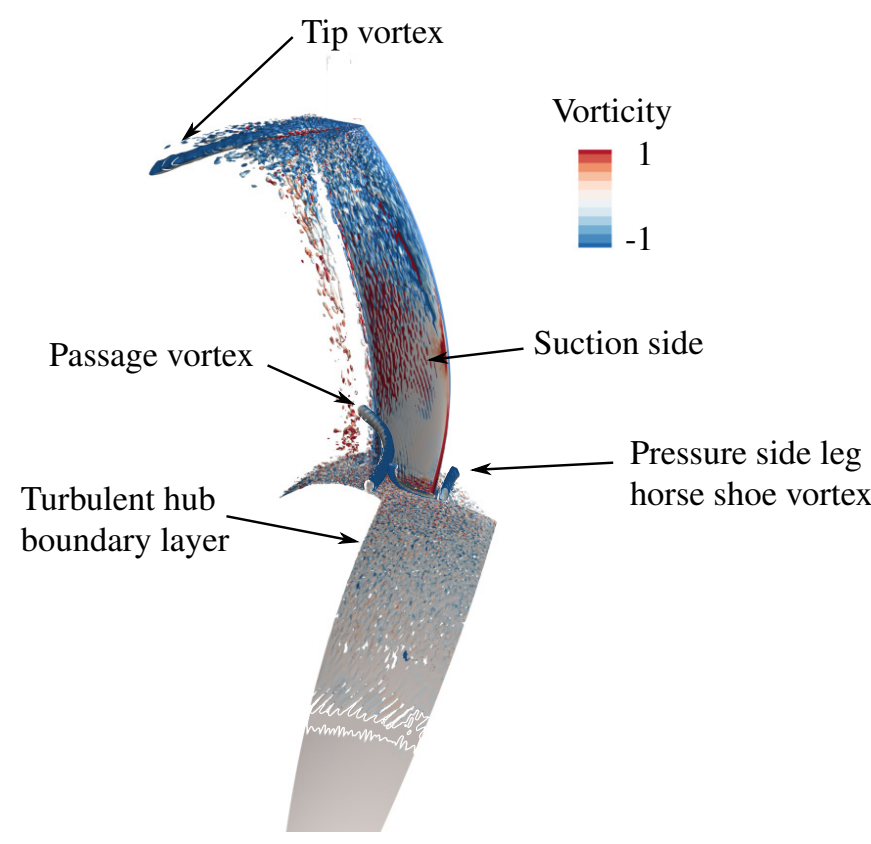

Fig. 19: Iso-contour of Q-criterion colored by the vorticity magnitude

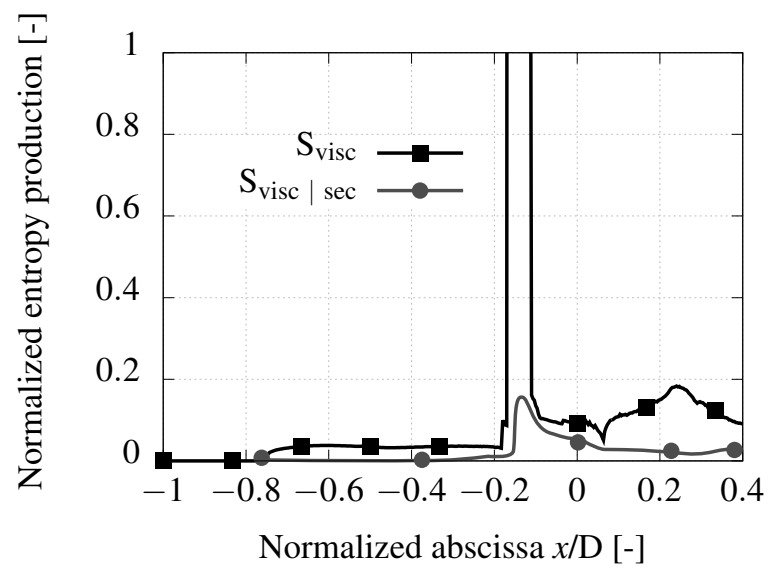

Fig. 20: Total viscous entropy production and contribution related to secondary vortices

the leading edge. The wake in all simulation was larger compared to the experiments and attributed to a thicker boundary layer at the blade trailing edge.

The analysis of the losses generated in the configuration has been led based on the LES simulation and an entropy formulation. The results indicate that the blade boundary layer is the main contribution to losses. The nacelle boundary layer and the contribution out of the boundary layers related to the passage, wake and tip vortices are of a lower magnitude in the generation of losses.

\section{Acknowledgments}

The authors are thankful to Airbus Operation SAS for technical support and experimental data provided, DLR for PIV experimental measurements, the European commission for having funded this research project within the project SCONE (Grant agreement ID: 755543), to ONERA for licensing Cerfacs to use code elsA (ONERA-Airbus-Safran property). Numerical post-processing have been performed using the free python-based Library Antares. Part of this work was performed using HPC resources from GENCI[CCRT-CINES-IDRIS] (Grant 2018-[A0042A06074]).

\section{Nomenclature}

Latin letters:

$\begin{array}{ll}c & \text { speed of sound } \\ C_{x} & \text { axial chord-length } \\ C_{p} & \text { pressure coefficient } \\ C_{\mathrm{M}}, C_{\mathrm{T}} & \text { mass flow, thrust coefficient } \\ \mathrm{D} & \text { rotor diameter } \\ f & \text { frequency } \\ F_{T} & \text { thrust } \\ H & \text { shape factor } \\ k & \text { turbulent kinetic energy } \\ M a & \text { inlet Mach number } \\ \dot{m} & \text { mass flow rate } \\ R e_{D} & \text { Reynolds number uD/ } / \\ S & \text { volumetric entropy generation } \\ T u & \text { turbulence intensity } \\ u & \text { velocity } \\ w & \text { memory data storage } \\ (x, y, z) & \text { cartesian coordinates } \\ y_{1} & \text { wall-normal characteristic width }\end{array}$

Greek letters:

$\delta \quad$ boundary layer thickness

$\lambda$ thermal conductivity

$\kappa \quad$ artificial viscosity coefficient

$\omega \quad$ rotational speed

$\mu \quad$ dynamic viscosity

$v \quad$ kinematic viscosity

$\theta \quad$ momentum thickness

$\tau \quad$ viscous stress tensor

Subscripts and superscripts:

$\infty \quad$ upstream condition

M.P. matching periodic simulation

P.L. phase-lagged simulation

RMS root mean square

tot total quantity

therm thermal

SGS subgrid scale contribution

visc viscous

.$^{+} \quad$ non-dimensional wall-units

. $\quad$ fluctuating quantity

Acronyms

CROR Counter Rotative Open Rotor

FSD Fourier Series Decomposition

LES Large-Eddy Simulation

POD Proper Orthogonal Decomposition

URANS Unsteady Reynolds Averaged Navier-Stokes 


\section{References}

[1] Schnell, R., Yin, J., Voss, C., and Nicke, E., 2012. "Assessment and Optimization of the Aerodynamic and Acoustic Characteristics of a Counter Rotating Open Rotor". Journal of Turbomachinery, 134(6), p. 061016.

[2] Van Zante, D. E., 2015. "Progress in open rotor research: A U.S. perspective". In Proceedings of the ASME Turbo Expo, ASME, pp. 120-131. Montreal, Quebec, Canada.

[3] Hendricks, E., and Tong, M., 2012. "Performance and Weight Estimates for an Advanced Open Rotor Engine". In 48th AIAA/ASME/SAE/ASEE Joint Propulsion Conference \& Exhibit, AIAA, pp. 148-160. Atlanta, Georgia.

[4] Ricouard, J., Julliard, E., Omais, M., Regnier, V., Parry, A., and Baralon, S., 2010. "Installation Effects on Contra-Rotating Open Rotor Noise". In 16th AIAA/CEAS Aeroacoustics Conference, AIAA, pp. 68-79. Atlanta, Georgia.

[5] Lepot, I., Leborgne, M., Schnell, R., Yin, J., Delattre, G., Falissard, F., and Talbotec, J., 2011. "Aeromechanical optimization of a contra-rotating open rotor and assessment of its aerodynamic and acoustic characteristics". In Proceedings of the Institution of Mechanical Engineers, Part A: Journal of Power and Energy.

[6] Carazo, A., Roger, M., and Omais, M., 2012. "Analytical Prediction of Wake-Interaction Noise in CounterRotating Open Rotors". In 17th AIAA/CEAS Aeroacoustics Conference (32nd AIAA Aeroacoustics Conference), AIAA, pp. 228-235. Portland, Oregon.

[7] Colin, Y., Caruelle, B., and Parry, A., 2012. "Computational strategy for predicting CROR noise at lowspeed Part III: investigation of noise radiation with the Ffowcs-Williams Hawkings analogy". In 18th AIAA/CEAS Aeroacoustics Conference (33rd AIAA Aeroacoustics Conference), AIAA, pp. 428-440. Colorado Springs.

[8] Colin, Y., Carazo, A., Caruelle, B., Node-Langlois, T., and Parry, A., 2012. "Computational strategy for predicting CROR noise at low-speed Part I: review of the numerical methods". In 18th AIAA/CEAS Aeroacoustics Conference (33rd AIAA Aeroacoustics Conference), AIAA, pp. 385-397. Colorado Springs.

[9] Colin, Y., Blanc, F., Caruelle, B., Barrois, F., and Djordjevic, N., 2012. "Computational strategy for predicting CROR noise at low-speed Part II: investigation of the noise sources computation with the chorochronic method". In 18th AIAA/CEAS Aeroacoustics Conference (33rd AIAA Aeroacoustics Conference), AIAA, pp. 322-331. Colorado Springs.

[10] Sagaut, P., 2006. Large Eddy Simulation for Incompressible Flows: An Introduction. Springer Science \& Business Media.

[11] Erdos, J., and Alzner, E., 1977. Computation of unsteady transonic flows through rotating and stationary cascades. 1: Method of analysis. Tech. rep., National Aeronautics and Space Administration.

[12] He, L., 1992. "Method of simulating unsteady turbo- machinery flows with multiple perturbations". AIAA Journal, 30(11), pp. 2730-2735.

[13] He, L., 2010. "Fourier methods for turbomachinery applications". Progress in Aerospace Sciences, 46(8), pp. 329-341.

[14] Berkooz, G., 2002. "The Proper Orthogonal Decomposition in the Analysis of Turbulent Flows". Annual Review of Fluid Mechanics, 25, pp. 539-575.

[15] Cambier, L., Heib, S., and Plot, S., 2013. "The Onera elsA CFD software: input from research and feedback from industry". Mechanics \& Industry, 14(3), pp. 159174.

[16] Mouret, G., Gourdain, N., and Castillon, L., 2015. "Adaptation of Phase-Lagged Boundary Conditions to Large Eddy Simulation in Turbomachinery Configurations". Journal of Turbomachinery, 138(4), p. 041003.

[17] Negulescu, C. A., 2013. "Airbus AI-PX7 CROR Design Features and Aerodynamics". SAE International Journal of Aerospace, 6(2), pp. 626-642.

[18] Novara, M., Geisler, R., and Schröder, A., 2015. "Multi-stereo PIV measurement of propeller wake flow in industrial facility". In 31st AIAA Aerodynamic Measurement Technology and Ground Testing Conference, pp. 55-68. Dallas, Texas.

[19] Piomelli, U., 2008. "Wall-layer models for large-eddy simulations". Progress in Aerospace Sciences, 44(6), pp. 437-446.

[20] Gourdain, N., Sicot, F., Duchaine, F., and Gicquel, L., 2014. "Large eddy simulation of flows in industrial compressors: a path from 2015 to 2035". Phil. Trans. R. Soc. A, 372(2022), p. 20130323.

[21] Pichler, R., Zhao, Y., Sandberg, R. D., Michelassi, V., Pacciani, R., Marconcini, M., and Arnone, A., 2018. "LES and RANS analysis of the end-wall flow in a linear LPT cascade with variable inlet conditions, Part I: Flow and secondary vorticity fields". In ASME Turbo Expo 2018: Turbomachinery Technical Conference and Exposition, ASME, pp. 532-544. Oslo, Norway.

[22] Tucker, P. G., 2011. "Computation of unsteady turbomachinery flows: Part 2-LES and hybrids". Progress in Aerospace Sciences, 47(7), pp. 546-569.

[23] Abu-Ghannam, B. J., and Shaw, R., 1980. "Natural Transition of Boundary LayersThe Effects of Turbulence, Pressure Gradient, and Flow History". Journal of Mechanical Engineering Science, 22(5), oct, pp. 213228.

[24] Casalino, D., Hazir, A., and Mann, A., 2017. "Turbofan Broadband Noise Prediction Using the Lattice Boltzmann Method". AIAA Journal, 56(2), pp. 132-143.

[25] Nishikawa, H., Rad, M., and Roe, P., 2013. "A thirdorder fluctuation splitting scheme that preserves potential flow". In 15th AIAA Computational Fluid Dynamics Conference, pp. 342-352. Anaheim, California.

[26] Wilcox, D. C., 2008. "Formulation of the k- $\omega$ turbulence model revisited". In AIAA Journal, Vol. 46, pp. 2823-2832.

[27] Jameson, A., Schmidt, W., and Turkel, E., 1981. 

nite volume methods using Runge-Kutta time-stepping schemes". AIAA paper, 6(2), p. 1259.

[28] Nicoud, F., and Ducros, F., 1999. "Subgrid-scale stress modelling based on the square of the velocity gradient tensor". Flow, Turbulence and Combustion, 62(3), pp. 183-200.

[29] Marmignon, C., Couaillier, V., and Courbet, B., 2011. "Solution Strategies for Integration of SemiDiscretized Flow Equations in elsA and CEDRE". AerospaceLab Journal, 2(1), pp. 1-11.

[30] Cliquet, J., Houdeville, R., and Arnal, D., 2008. “Application of Laminar-Turbulent Transition Criteria in Navier-Stokes Computations". AIAA Journal, 46(5), pp. 1182-1190.

[31] Cousteix, J., 1986. "Three-Dimensional and Unsteady Boundary-Layer Computations". Annual Review of Fluid Mechanics, 18(2), pp. 173-196.

[32] Schlichting, H., and Gersten, K., 2001. Boundary Layer Theory, Vol. 9.

[33] Adrian, R. J., 2005. "Twenty years of particle image velocimetry". Experiments in Fluids, 39(6), pp. 159169.

[34] Zlatinov, M. B., Sooi Tan, C., Montgomery, M., Islam, T., and Harris, M., 2012. "Turbine Hub and Shroud Sealing Flow Loss Mechanisms". Journal of Turbomachinery, 134(6), p. 061027.

[35] Michelassi, V., and Wissink, J. G., 2015. "Turbulent kinetic energy production in the vane of a low-pressure

linear turbine cascade with incoming wakes". International Journal of Rotating Machinery, 2015(2).

[36] Wheeler, A. P. S., Sandberg, R., Sandham, N. D., Pichler, R., Michelassi, V., and Laskowski, G., 2016. "Direct Numerical Simulations of a High-Pressure Turbine Vane". Journal of Turbomachinery, 138(7), p. 071003.

[37] Lengani, D., Simoni, D., Ubaldi, M., Zunino, P., Bertini, F., and Michelassi, V., 2017. "Accurate Estimation of Profile Losses and Analysis of Loss Generation Mechanisms in a Turbine Cascade". Journal of Turbomachinery, 139(12), pp. 121-132.

[38] Lengani, D., Simoni, D., Ubaldi, M., Zunino, P., and Bertini, F., 2017. "A POD-Based Procedure for the Split of Unsteady Losses of an LPT Cascade". International Journal of Turbomachinery, Propulsion and Power, 2(4), p. 17.

[39] Lengani, D., Simoni, D., Pichler, R., Sandberg, R. D., Michelassi, V., and Bertini, F., 2018. "Identification and quantification of losses in a LPT cascade by POD applied to LES data". International Journal of Heat and Fluid Flow, 70, pp. 28-40.

[40] Smith, L. H., 1987. "Unducted fan aerodynamic design”. Journal of Turbomachinery, 109(3), pp. 313324.

[41] Newmann, H. E., Bober, L. J., Serafini, J. S., and Li-Ko, C., 1983. "Analytical and experimental comparison of the flow field of an advanced swept turboprop". NASA Technical Memorandum, 11(3), pp. 122-133. 